ARTIGO DE REVISÃO

DÉBORA ARAÚJO MENDES VILELA

HIGOR BERMUDES NASCIMENTO

SÔNIA MARIA MOTTA PALMA

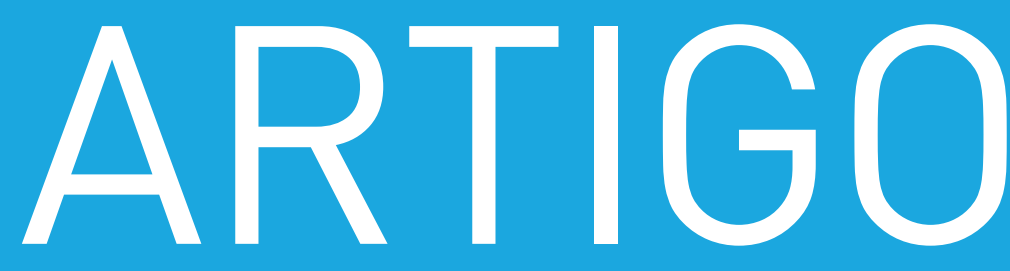

\title{
DISFUNÇÃO GASTROINTESTINAL NO TRANSTORNO DO ESPECTRO AUTISTA E SUAS POSSIVEIS CONDUTAS TERAPÊUTICAS
}

\section{GASTROINTESTINAL DYSFUNCTION IN AUTISTIC SPECTRUM DISORDER AND THERAPEUTIC POSSIBILITIES}

\section{Resumo}

O transtorno do espectro autista (TEA) é um distúrbio do neurodesenvolvimento definido por déficits sociais, deficiências de linguagem e comportamentos repetitivos com interesses restritos. Os distúrbios gastrointestinais são comuns em crianças com TEA, e os estudos sugerem que essas alterações podem interferir na patogênese e no prognóstico desses indivíduos. Os sintomas mais comuns são diarreia crônica, constipação, desconforto abdominal, refluxo gastroesofágico e intolerância alimentar. As disfunções gastrointestinais podem se manifestar apenas por alterações comportamentais e, assim, interferir no funcionamento do indivíduo, podendo também afetar o relacionamento familiar, sendo determinantes da qualidade vida desses indivíduos. Essas alterações podem se apresentar na forma de auto e heteroagressão, bem como de perturbação do sono ou irritabilidade. Em razão da dificuldade de interação social e da alteração na fala dos indivíduos com TEA, algumas vezes os distúrbios gastrointestinais não são reconhecidos pela equipe assistente, e as alterações comportamentais são exclusivamente atribuídas ao TEA, o que leva ao insucesso do tratamento. Os psicofármacos utilizados para tratamento das alterações comportamentais em portadores de TEA, como a risperidona, também podem contribuir para a mudança do padrão de alimentação dessas crianças e para as alterações gastrointestinais. É importante um acompanhamento multidisciplinar, a fim de otimizar e assegurar o diagnóstico mais precoce e identificar os problemas gastrointestinais, estabelecendo-se, assim, um tratamento individualizado que evite limitações futuras.

Palavras-chave: Distúrbios gastrointestinais, transtorno do espectro autista, dieta.

\section{Abstract \\ Autistic spectrum disorder (ASD) is a} neurodevelopmental disorder defined by social deficits, language impairments, and repetitive behaviors with restricted interests. Gastrointestinal disorders are common in children with ASD, and studies have suggested that these changes may interfere with pathogenesis and prognosis in these individuals. The most common symptoms are chronic diarrhea, constipation, abdominal discomfort, gastroesophageal reflux and food intolerance. Gastrointestinal dysfunctions may manifest only by behavioral changes and thus interfere with the functioning of the individual, and may also affect the family relationship, being determinants of quality of life in these individuals. These changes may manifest as self- and hetero-aggression, as well as sleep disturbance or irritability. Due to the social interaction difficulties and speech alterations observed in individuals with ASD, in some cases gastrointestinal disorders are not recognized by the medical team, and behavioral changes are exclusively attributed to ASD, leading to treatment failure. Psychopharmaceuticals used to treat behavioral changes in ASD patients, such as risperidone, may also contribute to altering these children's dietary patterns and gastrointestinal problems. Multidisciplinary followup is important in order to optimize and ensure earlier diagnosis and identify gastrointestinal problems, thus establishing individualized treatment and avoiding future limitations.

Keywords: Gastrointestinal disorders, autism spectrum disorder, diet. 


\section{DÉBORA ARAÚJO MENDES VILELA' ${ }^{1}$, HIGOR BERMUDES NASCIMENTO', SÔNIA MARIA MOTTA PALMA²}

${ }^{1}$ Médico residente, Serviço de Psiquiatria, Faculdade de Medicina, Universidade de Santo Amaro Santo Amaro, SP. ${ }^{2}$ Coordenadora, Ambulatório de Psiquiatria da Infância e Adolescência Faculdade de Medicina, Universidade de Santo Amaro, Santo Amaro, SP.

\section{INTRODUÇÃO}

Em 1943, o psiquiatra austríaco radicado nos Estados Unidos da América, Leo Kanner (1894-1981), criou o termo "autismo infantil" em seu ensaio clássico "Autistic disturbances of affective contact" (Transtornos autistas de contato afetivo) ${ }^{1}$. O transtorno do espectro autista (TEA) é um distúrbio do neurodesenvolvimento definido, segundo os critérios do Manual Diagnóstico e Estatístico de Transtornos Mentais, $5^{a}$ edição (DSM-5), por déficits sociais, deficiências de linguagem e comportamentos repetitivos com interesses restritos ${ }^{2}$. $\bigcirc$ gênero masculino é o mais afetado, sendo a prevalência na proporção de 4:2 para o gênero feminino; porém, nas mulheres, os sintomas podem se apresentar de forma mais intensificada ${ }^{3}$.

No Brasil, os dados epidemiológicos são insuficientes, mas se estima uma prevalência de aproximadamente 500.000 pessoas com TEA, com base no censo de 2000; porém, ao se considerar a estimativa internacional, esse número ultrapassa 1,5 milhão de pessoas ${ }^{4}$.

Em nossa prática clínica, observamos muitas queixas do trato gastrointestinal (TGI) em crianças com o diagnóstico de TEA, o que vem ao encontro de estudos recentes envolvendo o eixo intestino-cérebro e nos motivou a elaborar esta revisão integrativa buscando respaldo na literatura.

Estudos propõem que as alterações gastrointestinais podem ter influência na patogênese e prognóstico do TEA ${ }^{5}$. Evidências crescentes sugerem que a microbiota é alterada em resposta a fatores de risco etiológicos para TEA. A infecção materna é o principal fator de risco ambiental, com base em numerosos aspectos epidemiológicos, clínicos e estudos em animais. Modelar a ativação imune materna em camundongos resulta em alterações na composição do microbioma da prole adulta. Essa disbiose microbiana está correlacionada com anormalidades comportamentais duradouras, neuropatologias, disfunção imune e integridade gastrointestinal deficiente ${ }^{6}$. A porcentagem de indivíduos que sofrem de problemas gastrointestinais é variável de estudo para estudo, mas altas taxas foram relatadas, sendo os sintomas mais comuns diarreia crônica, constipação, desconforto abdominal, refluxo gastroesofágico e intolerância alimentar ${ }^{5}$.

As alterações gastrointestinais são desagradáveis e podem levar à diminuição da capacidade de concentração, frustração, problemas comportamentais e possivelmente hetero e autoagressão, especialmente em crianças incapazes de se comunicar. Crianças com dor abdominal são mais propensas a apresentar distúrbios psicológicos, ansiedade, problemas comportamentais ou outros sintomas psicológicos?.

Muitos estudos relataram o uso de antibiótico oral significativamente maior em crianças com autismo do que em outras crianças. Esses antibióticos podem perturbar a microbiota protetora que desempenha um papel importante na função gastrointestinal normal. 0 rompimento dessa microbiota intestinal normal poderia criar condições favoráveis paraa colonização por patógenos e/ou bactérias nocivas e, como hipótese, poderia causar ou contribuir para a disfunção gastrointestinal comumente relatada em populações com TEA ${ }^{8}$.

O presente estudo tem como objetivo ampliar o nosso conhecimento sobre o assunto, correlacionando-o com a nossa prática clínica.

\section{MÉTodos}

O presente estudo é uma revisão integrativa que visa explorar a hipótese de participação das alterações gastrointestinais na patogênese do TEA e no seu prognóstico. A pesquisa bibliográfica foi realizada nas plataformas eletrônicas PubMed, MEDLINE e SciELO, utilizando os seguintes descritores: (autism spectrum disorder OR autistic disorder OR autismo OR developmental disorders) AND (gastrointestinal disorders OR gastrointestinal symptoms OR gastrointestinal system OR microbiota) AND (diet supplementation OR diet OR dietary supplementation and treatment OR immune system diseases) OR problem behavior. Foram selecionados artigos publicados entre os anos de 2008 e 2019. Os seguintes critérios de inclusão foram considerados: artigos em português e inglês; estudos prospectivos e retrospectivos observacionais, estudos de caso-controle, estudos de coorte ou revisão sistêmica; artigos que investigaram perfis de microbiota intestinal e seus metabólitos em crianças com TEA e estudos incluindo informações sobre intervenção nutricional e estado nutricional em pacientes com TEA. Todos os estudos que não se enquadraram nos critérios mencionados foram excluídos do processo de revisão. Selecionamos 15 artigos que atendiam à proposta deste 


\section{ARTIGO DE REVISÃO}

DÉBORA ARAÚJO MENDES VILELA

HIGOR BERMUDES NASCIMENTO

SÔNIA MARIA MOTTA PALMA

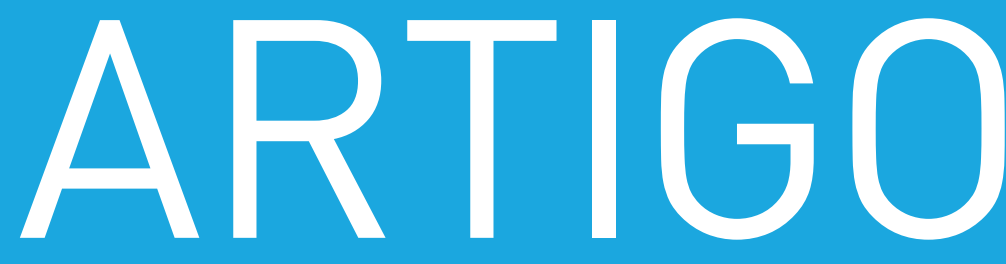

estudo e, em seguida, foi realizada uma síntese narrativa dos resultados obtidos (Figura 1).

\section{Resultados}

Em 2009, Ibrahim et al. publicaram um estudo com o objetivo de determinar se crianças com autismo têm uma incidência aumentada de sintomas gastrointestinais em comparação com controles pareados em uma amostra populacional. Foram incluídas 124 crianças com diagnóstico de autismo. Houve diferenças significativas entre os casos de TEA e controles na incidência de constipação e seletividade alimentar, porém não houve associação significativa entre o autismo e a incidência geral de sintomas gastrointestinais?

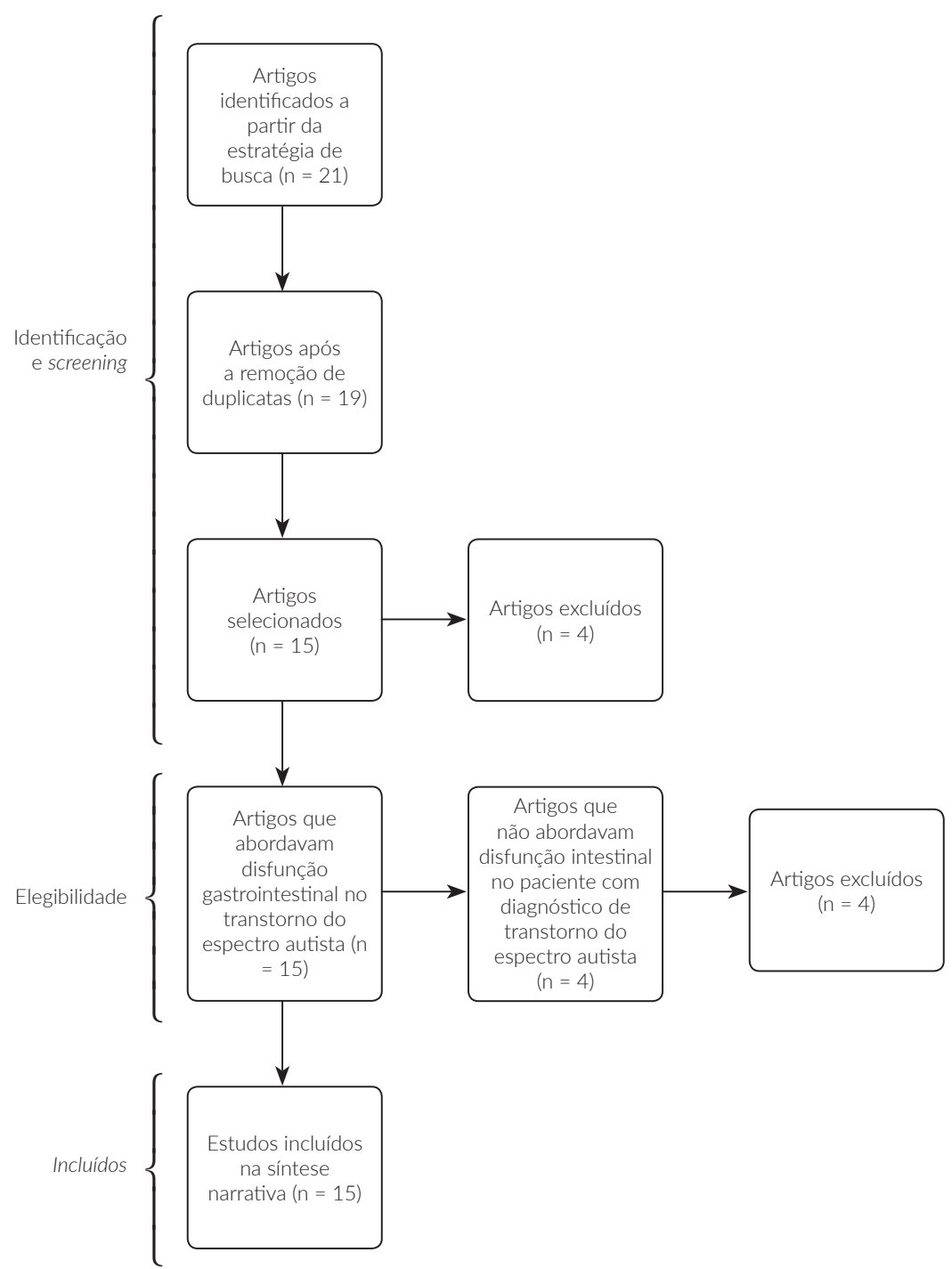

Figura 1 - Estratégia de busca segundo o Preferred Reporting Items for Systematic Reviews and Meta-Analyses (PRISMA). 


\section{DÉBORA ARAÚJO MENDES VILELA', HIGOR BERMUDES NASCIMENTO', SÔNIA MARIA MOTTA PALMA²}

${ }^{1}$ Médico residente, Serviço de Psiquiatria, Faculdade de Medicina, Universidade de Santo Amaro Santo Amaro, SP. 2 Coordenadora, Ambulatório de Psiquiatria da Infância e Adolescência, Faculdade de Medicina, Universidade de Santo Amaro, Santo Amaro, SP.

Em 2013, Kawicka \& Regulska-Ilow publicaram sobre a importância da terapia nutricional em indivíduos com TEA e como uma dieta apropriada pode ajudar a aliviar a gravidade da doença. A revisão da literatura demonstrou que a patogênese do autismo pode já ter um começo na vida fetal e durante a gravidez, e deve-se avaliar adequadamente o estado nutricional das gestantes antes e durante a gravidez, permitindo alterações na nutrição sempre que necessário³.

Em uma revisão bibliográfica em 2011, Pinho et al. demonstraram como a presença de alterações gastrointestinais e imunológicas nos pacientes portadores de TEA impactam na qualidade de vida da criança e da família, e reforçaram a necessidade de uma abordagem multidisciplinar dessas crianças 5 .

Wasilewska \& Klukowski, em 2015, corroboraram a contribuição do eixo intestino-cérebro para alterações no comportamento e cognição. Foi demonstrado que a desregulação do microbioma intestinal está envolvida na modulação das funções gastrointestinais, adquirindo a capacidade de afetar a permeabilidade intestinal, a função imune da mucosa e a motilidade e a sensibilidade intestinal?.

Buie et al., em 2010, reforçaram a necessidade de novos estudos com embasamento científico para a avaliação diagnóstica e o tratamento de problemas gastrointestinais nessa população de pacientes. Porém, a opinião de especialistas e o relato de pais de crianças autistas evidenciam melhorias nos comportamentos problemáticos por meio de intervenções nutricionais ou médicas ${ }^{10}$.

Já Cubala-Kurcharska publicou, em 2010, um estudo em que foram realizadas 500 endoscopias e biópsias em crianças com TEA, demonstrando que a inflamação crônica do trato digestivo era prevalente e parecia ter um grande papel no agravamento da condição do austista ${ }^{11}$. Corroborando o estudo de Cubala-Kurcharska, Adams et al. publicaram um estudo em 2011 que avaliou a flora gastrointestinal a partir de amostras de fezes de 58 crianças com TEA e 39 crianças do grupo de controle. Esse estudo sugeriu fortemente que problemas gastrointestinais estão associados à gravidade do autismo $^{12}$. O estudo de Pessoa et al. e Ristori et al. publicados em 2014 e 2019, respectivamente, também reforçam essa associação ${ }^{13,14}$.
Um estudo transversal conduzido em 2014 por Tschinkel \& Consolo tentou correlacionar o TEA com deficiências de mineirais e evidenciou que a concentração de selênio é reduzida em autistas, porém não houve correlação entre o estado nutricional e a concentração dos elementos ${ }^{4}$.

Campbell et al., em 2009, associaram a sinalização interrompida do gene MET ao aumento do risco de TEA e de disfunção gastrointestinal familiar ${ }^{8}$.

Para Vuong \& Hsiao, a modulação do ambiente materno também é de interesse, dadas as origens do desenvolvimento neurológico do TEA. Embora existam vários fatores de risco perinatais que influenciam a fisiologia materno-fetal, incluindo estresse, infecção, diabetes gestacional, aleitamento materno versus fórmula, idade materna, uso de antibióticos e obesidade, as alterações na microbiota intestinal também podem ser um fator de risco relevante ${ }^{6}$.

Abdelrahman et al. hipotetizaram que polimorfismos genéticos relacionados à serotonina podem se associar ao aparecimento de alterações comportamentais relacionadas ao autismo e às alterações gastrointestinais associadas nesses pacientes ${ }^{15}$.

Para Ng et al., existem evidências gerais limitadas para apoiar o papel dos prebióticos ou probióticos no alívio dos sintomas gastrointestinais ou comportamentais em crianças com TEA ${ }^{16}$.

\section{DISCUSSÃO}

O TEA, que antes era visto como uma doença intratável unicamente no cérebro, é agora considerado uma disfunção do sistema nervoso central acompanhada de desordens do corpo em diferentes órgãos e sistemas, como no sistema imunológico ou no trato digestivo. 0 polimorfismo genético está associado à variabilidade da apresentação clínica do TEA e aos problemas comórbidos?.

Abdelrahman et al. evidenciaram em seu estudo que a frequência do genótipo AA do polimorfismo -1438A/ $G$ do gene HTR2A e a frequência do genótipo CC do polimorfismo 102T/C aumentam significativamente em indivíduos com TEA e disfunção gastrointestinal em comparação com os pacientes com TEA sem alteração gastrointestinal $(31,3 \text { versus 6,3\% })^{15}$. 


\section{ARTIGO DE REVISÃO}

DÉBORA ARAÚJO MENDES VILELA HIGOR BERMUDES NASCIMENTO SÔNIA MARIA MOTTA PALMA

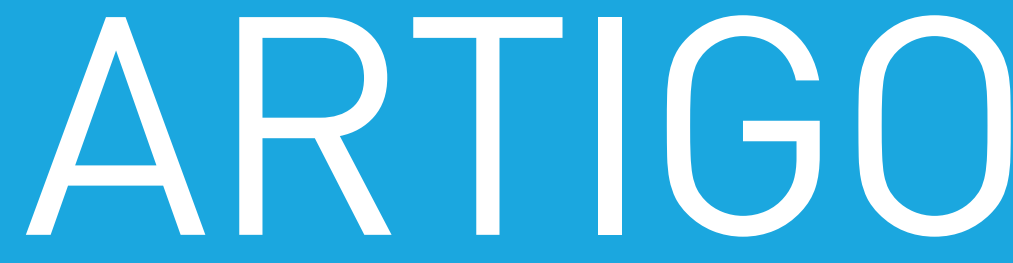

A vulnerabilidade genética e interações gene-ambiente contribuem para o risco desse transtorno. Em 2009, foi descrita por Campbell et al. uma associação genética do TEA com uma variante funcional no promotor 5 do gene que codifica o receptor MET tirosina-quinase. Essa variante é mais comum em indivíduos com TEA do que em amostras gerais da população humana. Como um receptor pleiotrópico de tirosina-quinase, o MET também participa da função imune e gastrointestinal. Se o MET estiver alterado nesses sistemas em indivíduos com TEA, a predição é de que a sinalização MET interrompida poderá contribuir para distúrbios comportamentais e gastrointestinais ${ }^{8}$.

A serotonina é considerada uma parte vital do sistema de comunicação bidirecional entre o cérebro e o intestino. A perturbação do sistema serotoninérgico tem sido implicada na etiologia do TEA e em vários distúrbios neuropsiquiátricos. Portanto, levanta-se a hipótese de que polimorfismos de genes relacionados à serotonina podem se associar ao aparecimento de características do comportamento autista e à disfunção gastrointestinal associada nesses pacientes ${ }^{15}$.

Recentemente, um estudo envolvendo 58 crianças com TEA, medindo os sintomas gastrointestinais e a gravidade do autismo, mostrou uma forte associação positiva de severidade do autismo e disfunção gastrointestinal. Essa associação foi evidente dentro de cada uma das subcategorias de avaliação do autismo, incluindo fala, interação social, função sensorial e cognitiva, e habilidade física e comportamental. Apesar de não definir uma relação de causa e efeito, esse estudo enfatiza a importância do desenvolvimento de novas abordagens para aliviar sintomas gastrointestinais em crianças com autismo ${ }^{13}$.

Um mecanismo imune ou mediado por inflamação, específico para TEA, acarretando aumento da permeabilidade do intestino, tem sido hipotetizado para explicar os distúrbios gastrointestinais observados em indivíduos com TEA, principalmente com base no achado de hiperplasia linfoide nodular ileal e/ou colite crônica observado em colonoscopias. Contudo, achados semelhantes são conhecidos por estarem presentes em crianças com desenvolvimento típico, bem como crianças com alergias alimentares e imunodeficiências. $\bigcirc$ significado desses achados, portanto, não está claro ${ }^{10}$.
Sintomas subjetivos do TGI, como dor, desconforto, azia ou náusea, são muito difíceis de avaliar e interpretar devido aos principais sintomas do TEA, como dificuldades na comunicação verbal e não verbal, bem como uma percepção alterada da dor. Expressões faciais, como caretas, ranger de dentes, mastigação excessiva (de alimentos ou itens), são frequentemente observadas na presença dos sintomas gastrointestinais. Comportamentos vocais de acompanhamento, como soluços, gritos ou ecolalia tardia, também podem estar presentes. Além disso, comportamentos motores, como a criança colocar pressão sobre o estômago com suas próprias mãos ou objetos, incluindo cadeiras ou mesas, podem estar associados a sintomas na área abdominal e são comumente relatados pelos pais e/ou cuidadores?.

A associação entre TEA e sintomas gastrointestinais leva a maiores riscos de alterações comportamentais quando esses pacientes são comparados a outros com TEA que não apresentam sintomas gastrointestinais. As alterações comportamentais interferem no funcionamento do indivíduo, podendo também afetar o relacionamento familiar, e são determinantes da qualidade vida. Essas alterações podem se manifestar na forma de auto e heteroagressão, bem como de perturbação do sono ou irritabilidade ${ }^{10}$. Os problemas de sono são comuns, incluem dificuldade em adormecer e dificuldade em permanecer no sono e foram encontrados em 44 a 83\% das crianças com TEA. A privação do sono pode ter consequências significativas para a saúde e pode piorar a função cognitiva, exercendo uma ação inibitória sobre a proliferação de neurônios no sistema nervoso central. Os desequilíbrios neuroendócrinos causados por distúrbios do sono são comparáveis às consequências da exposição crônica ao estresse?.

As alterações comportamentais mencionadas acima podem indicar desconforto abdominal. Por sua vez, o desconforto abdominal, bem como outros sintomas (constipação, flatulência e diarreia), podem ser manifestações de desregulação neuroentérica ou da síndrome do intestino irritável. Em um diagnóstico de exclusão, a síndrome do intestino irritável é difícil de distinguir de outras condições subjacentes sem que se façam testes invasivos. Nenhuma diretriz com base em evidências está disponível para orientar a avaliação desses sintomas. Por essa razão, médicos de 


\section{DÉBORA ARAÚJO MENDES VILELA', HIGOR BERMUDES NASCIMENTO', SÔNIA MARIA MOTTA PALMA²}

1 Médico residente, Serviço de Psiquiatria, Faculdade de Medicina, Universidade de Santo Amaro, Santo Amaro, SP. ${ }^{2}$ Coordenadora, Ambulatório de Psiquiatria da Infância e Adolescência, Faculdade de Medicina, Universidade de Santo Amaro, Santo Amaro, SP.

cuidados primários, psiquiatras, psicólogos, pediatras, e gastroenterologistas precisam trabalhar em conjunto para aprimorar a avaliação e o tratamento de sintomas gastrointestinais em indivíduos com TEA ${ }^{10}$.

A avaliação diagnóstica de distúrbios gastrointestinais com manifestações comportamentais em pacientes com TEA pode ser muito complexa e inclui história médica, exame físico, exames laboratoriais, exames de imagem/ radiológicos, estudos funcionais e endoscopia. Métodos menos invasivos são recomendados antes de qualquer internação planejada. Um relatório de consenso recomenda que as avaliações diagnósticas iniciais de sintomas e distúrbios gastrointestinais em indivíduos com TEA sejam escolhidas de acordo com o tipo de sintoma, conforme sintetizado na Tabela $1^{7}$.

Deve ser sempre realizado um histórico detalhado (incluindo história pessoal de doença alérgica, história alimentar e história familiar) para identificar possíveis comorbidades alérgicas. Modificações dietéticas, como remoção de leite para sintomas de intolerância à lactose, podem ser abordadas empiricamente, como com qualquer outro paciente pediátrico com sintomas consistentes. Os dados sobre o valor de dietas específicas eficazes no tratamento de indivíduos com TEA são difíceis de avaliar. Acredita-se que muitas modificações dietéticas tenham um resultado benéfico, embora os efeitos do placebo sejam provavelmente altos nesse cenário. Existem poucos estudos na literatura sobre o assunto e com grupos de controle adequados ${ }^{10}$.

É sabido que coexistimos com uma vasta população de espécies microbianas que habitam o corpo humano. É estimado que mais de 100 trilhões de células microbianas nos usam como hospedeiros, e provavelmente superam as células do corpo humano por uma ordem de magnitude, levando alguns a chamá-los de nosso "segundo genoma". Essa população microbiana participa da homeostase e, quando a balança se inclina para longe do microbioma saudável, pode haver um resultado negativo para a saúde humana ${ }^{11}$.

Acredita-se que bebês humanos nasçam com o TGI estéril, mas imediatamente após o nascimento, a colonização bacteriana do intestino se inicia. O modo do nascimento (parto vaginal ou cesáreo) exerce a primeira influência no desenvolvimento do microbioma infantil. Adicionalmente, a amamentação ao seio ou amamentação com formulações de leite industrializado leva a diferentes trajetórias no desenvolvimento da microbiota intestinal. No primeiro ano de vida, a microbiota intestinal está em constante mudança, ficando semelhante à adulta aos três anos de idade ${ }^{11}$

Tabela 1 - Procedimentos de diagnóstico inicial para crianças com transtorno do espectro autista, dependendo dos distúrbios gastrointestinais clínicos

\begin{tabular}{ll}
\hline Manifestação clínica do trato gastrointestinal & Procedimento diagnóstico inicial \\
\hline Sintomas gerais & Radiografia abdominal \\
Refluxo gastroesofágico & - Tratamento empírico com um inibidor da bomba de prótons por 2 a 4 \\
& semanas \\
& - Revisão da dieta \\
& - Elevação da cabeceira da cama ao dormir
\end{tabular}

Constipação intestinal

- Tratamento empírico com polietilenoglicol (PEG 3350) por 4 semanas

- Suplementação dietética com frutas, fibras e líquidos

Intolerância à lactose

Teste respiratório para pesquisar eliminação de hidrogênio no ar expirado

Diarreia

Análise de sangue oculto nas fezes, patógenos entéricos, parasitas (Giardia lamblia ou Cryptosporidium parvum) e Clostridium difficile 


\section{ARTIGO DE REVISÃO}

DÉBORA ARAÚJO MENDES VILELA HIGOR BERMUDES NASCIMENTO SÔNIA MARIA MOTTA PALMA

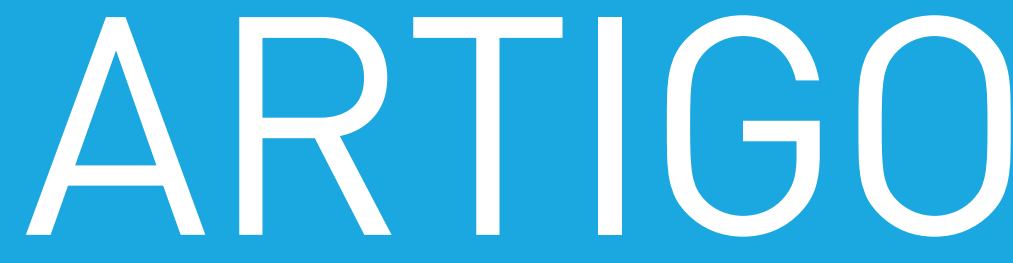

A descoberta de que diferentes composições microbianas estão associadas a alterações comportamentais e da cognição contribuiu significativamente para o estabelecimento do eixo microbiota-intestino-cérebro como uma extensão do bem-aceito conceito do eixo intestino-cérebro. A comunicação bidirecional intestinal-cerebral envolve diferentes vias e elementos da rede neural entérica. Esse eixo intestinal modula o metabolismo e o comportamento do corpo participando da regulação da homeostase de apetite e energia, resposta ao estresse, emoções e atitudes, aprendizado, memória e resposta à dor?.

O microbioma humano é dominado por dois filos, o Firmicutes e o Bacteroidetes, que correspondem respectivamente a aproximadamente 75 e 20\% da microbiota intestinal, com menores contribuições de Proteobacteria e Actinobacteria. $O$ crescimento excessivo de organismos anaeróbicos obrigatórios, como Clostridium spp (Firmicutes), bem como Desulfovibrio spp, e uma diminuição significativa na relação Bacteroidetes/ Firmicutes foram encontrados em crianças com autismo?.

Descobriu-se que o ácido propiônico apresenta-se elevado em amostras de fezes ou urina de crianças com TEA. Doenças conhecidas por estarem associadas a níveis elevados de ácidos graxos de cadeia curta (AGCC) incluem condições hereditárias e adquiridas, como acidemia propiônica/metilmalônica, deficiência de biotinidase/holocarboxilase e distúrbios mitocondriais. Essas doenças apresentam atraso no desenvolvimento, regressão, distúrbios convulsivos, bem como sintomas gastrointestinais. Um subgrupo de crianças com autismo com níveis elevados de AGCC pode se beneficiar de uma dieta pobre em carboidratos, reduzindo a produção de AGCC?.

Há um uso significativamente maior de antibiótico oral em crianças autistas, podendo sugerir alterações no sistema imune e da microbiota intestinal dessas crianças. Com a perda da flora normal, pode haver um supercrescimento da flora patogênica, causando problemas intestinais ${ }^{12}$.

Uma das características do TEA é uma disfunção da integração sensorial e sensibilidade sensorial, resultando em crianças com dietas autorrestritas, como evitação de alimentos de determinada cor ou consistência, hábitos alimentares seletivos ou comportamentos alimentares desordenados que podem levar a problemas secundários no estado nutricional da criança. Estudos que investigam a qualidade nutricional de crianças com autismo foram compilados concisamente em uma publicação de Coury et al., de 2012. As dietas recomendadas de energia, carboidratos, gorduras, proteínas e micronutrientes podem não ser satisfeitas, ou o nível de um nutriente pode estar elevado em comparação com outros. Ingestões inadequadas de vitamina D, vitamina A e cálcio foram mais comuns em crianças com TEA que em crianças saudáveis, o que reforça a necessidade de um acompanhamento multidisciplinar?

Muitas crianças com autismo são tratadas com dietas restritivas, vitaminas, minerais e outros suplementos alimentares, além de vários medicamentos voltados para possíveis distúrbios gastrointestinais. Esses tratamentos não devem ser fornecidos indiscriminadamente a crianças com autismo, a menos que haja evidência explícita da presença de um distúrbio gastrointestinal em um caso específico?.

Mais estudos são necessários para avaliar o efeito de diferentes intervenções dietéticas (como a dieta mediterrânea) nos sintomas gastrointestinais e, consequentemente, como tais intervenções podem afetar os padrões comportamentais associados aos distúrbios de TEA ${ }^{14}$.

No que diz respeito à suplementação com probióticos, há evidências gerais limitadas para apoiar a eficácia dos probióticos no alívio dos sintomas gastrointestinais ou das alterações comportamentais em crianças com TEA. O mecanismo exato pelo qual os probióticos exercem potenciais efeitos terapêuticos não é conhecido, mas existem várias hipóteses de que eles ajudariam a restaurar a microbiota intestinal de volta aos níveis normais, melhorando os sintomas gastrointestinais concomitantes. Na grande maioria dos estudos disponíveis, o tamanho da amostra foi limitado. Além disso, muitos desses estudos eram ensaios abertos e tinham como base questionários e pesquisas qualitativos e autorreferidos para avaliar a resposta ao tratamento, convidando potencial viés para os estudos. Também pode haver várias dificuldades experimentadas pelos pais na avaliação desses aspectos, principalmente devido aos déficits de comunicação típicos de crianças com TEA. Estudos randomizados e controlados, com uma população maior e o uso de 


\section{DÉBORA ARAÚJO MENDES VILELA', HIGOR BERMUDES NASCIMENTO', SÔNIA MARIA MOTTA PALMA²}

1 Médico residente, Serviço de Psiquiatria, Faculdade de Medicina, Universidade de Santo Amaro, Santo Amaro, SP. ${ }^{2}$ Coordenadora, Ambulatório de Psiquiatria da Infância e Adolescência, Faculdade de Medicina, Universidade de Santo Amaro, Santo Amaro, SP.

pontuação clínica, podem levar a estudos e resultados mais robustos ${ }^{16}$.

Os estudos aqui analisados são promissores, porém é importante salientar que são necessários mais estudos para validar essa associação entre a composição da flora intestinal e o desenvolvimento do TEA. Ainda é cedo para sabermos se serão geradas intervenções seguras que melhorem a qualidade de vida desses pacientes.

\section{CONCLUSÃo}

Os distúrbios gastrointestinais são comuns em crianças com TEA e podem contribuir para as alterações comportamentais desses indivíduos. Algumas vezes, os distúrbios gastrointestinais não são reconhecidos pela equipe assistente, e as alterações comportamentais são exclusivamente atribuídas ao TEA, o que leva ao insucesso do tratamento.

O pediatra deve estar atento às curvas de crescimento e desenvolvimento, e deve haver também um acompanhamento especializado e multidisciplinar a fim de otimizar e assegurar o diagnóstico precoce, tanto do TEA como da possível alteração gastrointestinal, e, desse modo, iniciar-se o tratamento individualizado adequado para que, no futuro, haja menos limitações.

Também é necessária a conscientização dos pais e cuidadores, pois muitas vezes essas crianças apresentam dificuldade de comunicação e nem sempre são capazes de localizar ou expressar seus sintomas de forma clara, fazendo-se necessário um exame físico detalhado. Destaca-se também a importância de protocolos para evitar exames invasivos de forma desnecessária.

Apesar das evidências científicas atualmente disponíveis, são necessários mais estudos abordando a associação entre TEA e distúrbios do TGl.

Artigo submetido em 10/12/2019, aceito em 24/01/2020. Os autores informam não haver conflitos de interesse associados à publicação deste artigo.

Fontes de financiamento inexistentes.

Correspondência: Sônia Maria Motta Palma, Universidade de Santo Amaro Campus I, Rua Professor Enéas de Siqueira Neto, 340, Jardim das Imbuias, CEP 04829-300, São Paulo, SP.

E-mail: soniammpalma@hotmail.com

\section{Referências}

1. Tamanaha AC, Perissinoto J, Chiari BM. Uma breve revisão histórica sobre a construção dos conceitos do autismo infantil e da síndrome de Asperger. Rev Soc Bras Fonoaudiol. 2008;13:296-9.

2. Associação Americana de Psiquiatria. Manual Diagnóstico e Estatístico de Transtornos Mentais, $5^{a}$ edição (DSM-5). Porto Alegre: Artmed; 2014.

3. KawickaA, Regulska-IlowB. Hownutritional status, diet and dietary supplements can affect autism. A review. Rocz Panstw Zakl Hig. 2013;64:1-12.

4. Tschinkel PFS, Consolo LZZ. Avaliação das concentrações plasmáticas dos elementos traço cobre, zinco e selênio em crianças com transtorno do espectro autista [dissertação]. Campo Grande: Universidade Federal de Mato Grosso do Sul; 2014.

5. Pinho MA, Silva LR. Manifestações digestórias em portadores de transtornos do espectro autístico necessidade de ampliar as perguntas e respostas. R Ci Med Biol. 2011;10:304-9.

6. Vuong HE, Hsiao EY. Emerging roles for the gut microbiome in Autism spectrum disorder. Biol Psychiatry. 2017;81:411-23.

7. Wasilewska J, Klukowski M. Gastrointestinal symptoms and autism spectrum disorder: links and risks - a possible new overlap syndrome. Pediatric Health Med Ther. 2015;6:153-66.

8. Campbell DB, Buie TM, Winter H, Bauman M, Sutcliffe JS, Perrin JM, et al. Distinct genetic risk based on association of MET in families with cooccurring autism and gastrointestinal conditions. Pediatrics. 2009;123:1018-24.

9. Ibrahim SH, Voigt RG, Katusic SK, Weaver AL, Barbaresi WJ. Incidence of gastrointestinal symptoms in children with autism: populationbased study. Pediatrics. 2009;124; 680-6.

10. Buie T, Campbell DB, Fuchs GJ 3rd, Furuta GT, Levy J, Vandewater J, et al. Evaluation, diagnosis, and treatment of gastrointestinal disorders in individuals with ASDs: a consensus report. Pediatrics. 2010;125 Suppl 1:S1-18.

11. Cubala-Kucharska M. The review of most frequently occurring medical disorders related 


\section{ARTIGO DE REVISÃO}

\section{DÉBORA ARAÚJO MENDES VILELA HIGOR BERMUDES NASCIMENTO SÔNIA MARIA MOTTA PALMA}

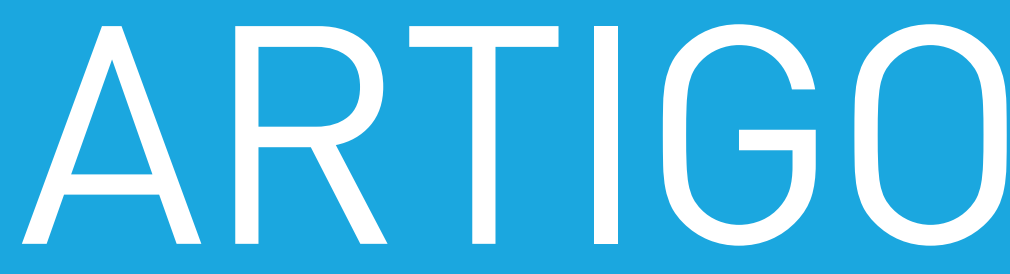

to etiology of autism and methods of treatment. Acta Neurobiol Exp (Wars). 2010;70:141-6.

12. Adams JB, Johansen LJ, Powell LD, Quig D, Rubin RA. Gastrointestinal flora and gastrointestinal status in children with autism--comparisons to typical children and correlation with autism severity. BMC Gastroenterol. 2011;11:22.

13. Pessoa Cm, Nunes RM. Transtorno do espectro do autismo e permeabilidade intestinal: influências da nutrição [monografia]. Juiz de fora: Faculdade de Nutrição, na Universidade Federal de Juiz de Fora; 2014.
14. Ristori MV, Quagliariello A, Reddel S, Ianiro G, Vicari S, Gasbarrini A, etal. Autism, gastrointestinal symptoms and modulation of gut microbiota by nutritional interventions. Nutrients. 2019;11:11.

15. Abdelrahman HM, Sherief LM, Alghobashy AA, Abdel Salam SM, Hashim HM, Abdel Fattah NR, et al. Association of 5-HT2A receptor gene polymorphisms with gastrointestinal disorders in Egyptian children with autistic disorder. Res Dev Disabil. 2015;36C:485-90.

16. Ng QX, Loke W, Venkatanarayanan N, Lim DY, Soh AY, Yeo Ws. A systematic review of the role of prebiotics and probiotics in autism spectrum disorders. Medicina (Kaunas). 2019;55:129. 